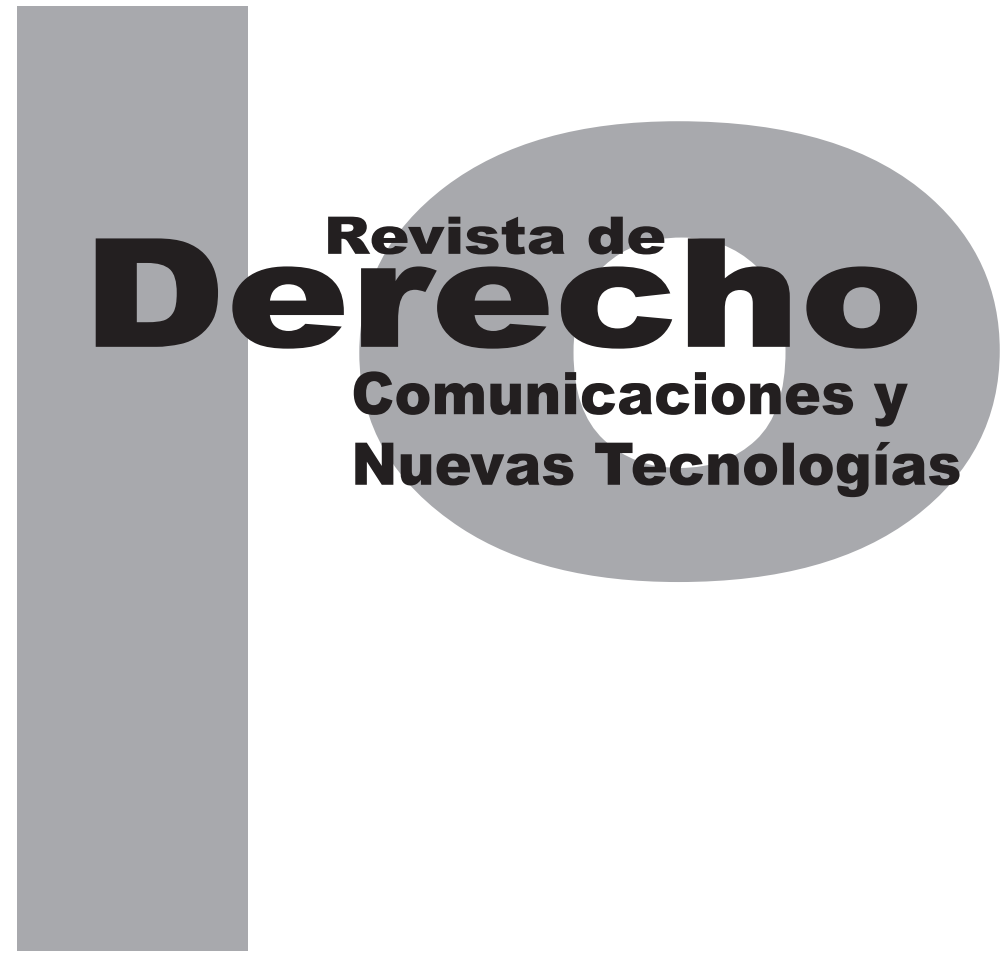

\title{
CONSOLIDACIÓN DE LA ECONOMÍA DIGITAL Y DESAFÍOS EN MATERIA DE PROTECCIÓN DE LA PRIVACIDAD
}

\author{
Guillermo Rodrigo CoRREdor CASTELLANOS
}

\author{
Artículo de reflexión
}

DOI: http://dx.doi.org/10.15425/redecom.14.2015.01

Universidad de los Andes

Facultad de Derecho

Rev. derecho comun. nuevas tecnol.

No. 14, julio - diciembre de 2015. ISSN 1909-7786 


\section{Consolidación de la economía digital y desafíos en materia de protección de la privacidad}

\section{Resumen}

El actual desarrollo que conocen las herramientas de procesamiento algorítmico de información está permitiendo cambios radicales en la forma como las empresas conciben y ejecutan sus estrategias de mercadeo y publicidad. Igualmente, está motivando un creciente interés en el análisis de los patrones de comportamiento de los usuarios de la red (behavioral targeting). En este contexto de innovaciones radicales y ante la necesidad de preservar el libre flujo de la información como medio fundamental para facilitar su acceso, almacenamiento y disponibilidad, se vienen proponiendo diferentes enfoques, los cuales, en su mayoría, implican una revisión de conceptos jurídicos fundamentales como el respeto a la privacidad, la libertad de expresión y el acceso a la información, poniendo de presente nuevos e interesantes matices para el ejercicio de los derechos fundamentales en el contexto de la denominada economía de los datos. El presente documento reconoce un papel destacado al Estado como actor llamado a equilibrar el gran poder e influencia adquirida por las empresas multinacionales en este sector, pero al mismo tiempo se centra en la importancia que tiene la inclusión de los conceptos desarrollados en el marco de la economía comportamental, y postula que a través de ellos es posible lograr regulaciones más coherentes y compatibles con los desafíos que enfrentan las economías emergentes.

Palabras clave: economía digital, privacidad, regulación, economía comportamental, países emergentes.

\section{Consolidation of the digital economy and challenges for the protection of privacy}

\section{Abstract}

The current development of tools used for the algorithmic processing of information is enabling radical changes in the way companies conceive and execute their marketing and advertising strategies. It is also encouraging a growing interest in the analysis of the behavioral patterns of network users (behavioral targeting). In this context of radical innovations and the need to preserve the free flow of information as a fundamental means for easy access, storage and availability, different approaches are being proposed, which mostly involve a review of basic legal concepts such as the respect for privacy, freedom of expression and access to information, thus presenting new and interesting nuances for the exercise of fundamental rights in the context of the so-called information economy. This paper recognizes a prominent role for the State to play as an actor to be called upon to balance the great power and influence acquired by multinational companies in this sector, but to also focus on the importance of the inclusion of the concepts developed in the behavioral economics framework, and to postulate that through this it will be possible to achieve regulations that are more consistent and compatible with the challenges faced by emerging market nations.

Keywords: digital economy, privacy, regulation, behavioral economics, emerging market nations.

\section{Consolidação da economia digital e desafios em matéria de proteção da privacidade}

\section{Resumo}

0 atual desenvolvimento que conhecem as ferramentas de processamento algorítmico de informação está permitindo mudanças radicais na forma como as empresas concebem e executam suas estratégias de mercado e publicidade. Igualmente, está motivando um crescente interesse na análise dos padrões de comportamento dos usuários da rede (behavioral targeting). Neste contexto de inovações radicais e ante a necessidade de preservar o fluxo livre da informação como meio fundamental para facilitar seu acesso, armazenamento e disponibilidade, se vêm propondo diferentes enfoques, os quais, em sua maioria, implicam uma revisão de conceitos jurídicos fundamentais como o respeito à privacidade, a liberdade de expressão e o acesso à informação, pondo de presente novos e interessantes matizes para o exercício dos direitos fundamentais no contexto da denominada economia dos dados. 0 presente documento reconhece um papel destacado ao Estado como ator chamado a equilibrar o grande poder e influência adquirida pelas empresas multinacionais neste setor, mas ao mesmo tempo se centra na importância que tem a inclusão dos conceitos desenvolvidos no marco da economia comportamental, e postula que através disso é possível conseguir regulações mais coerentes e compatíveis com os desafios que enfrentam as economias emergentes.

Palavras-chave: economia digital, privacidade, regulação, economia comportamental, países emergentes. 


\title{
Consolidación de la economía digital y desafíos en materia de protección de la privacidad*
}

\author{
Guillermo Rodrigo Corredor Castellanos ${ }^{* *}$
}

“Un hombre siempre y en todas partes, elige actuar como se le antoja y no como le dicen la razón y sus intereses; puesto que es muy probable que sienta deseos de actuar contra sus intereses."

Fiódor Dostoyevski. Memorias del subsuelo, 1864.

\section{SUMARIO}

Introducción - I. ESTADO ACTUAL DE LAS DISCUSIONES SOBRE REGULACIÓN DE LA PRIVACIDAD EN INTERNET - A. Rasgos principales de la economía digital - B. Actores de la economía digital - C. Problemas que surgen con el advenimiento y consolidación de la economía digital - II. PROPUESTAS REGULATORIAS EN TORNO A LA PRIVACIDAD - A. EI enfoque de la Federal Trade Commission (FTC) - B. El enfoque de la Unión Europea - C. Los enfoques de autorregulación - III. PERSPECTIVAS REGULATORIAS PARA COLOMBIA - A. Panorama general de las normas actuales - B. Espacio para la economía comportamental en la protección al derecho a la privacidad - C. La intervención del Estado en materia de big data - D. Acciones puntuales - IV. CONCLUSIONES - Referencias.

\footnotetext{
* Cómo citar este artículo: Corredor Castellanos, G. R. (Diciembre, 2015). Consolidación de la economía digital y desafíos en materia de protección de la privacidad. Revista de Derecho, Comunicaciones y Nuevas Tecnologías, 14. Universidad de los Andes (Colombia).

El presente artículo no ha contado con ninguna fuente de patrocinio financiero externo. Por lo tanto, las opiniones expresadas en él comprometen exclusivamente a su autor.

** Abogado y especialista en Propiedad Intelectual de la Universidad Externado de Colombia. Especialista en Gestión Pública e Instituciones Administrativas de la Universidad de los Andes. Máster en Derecho Económico Internacional de la Universidad de Berna (World Trade Institute). Actualmente es docente e investigador del Departamento de Derecho Económico de la Universidad Externado de Colombia. Correo: rodrigo.corredor@uexternado.edu.co
} 
Introducción

Las propuestas regulatorias en torno al concepto de economía digital o economía de los datos constituyen un tema principal dentro de la agenda económica de los países industrializados. En estos países, el desarrollo de las herramientas de tratamiento de información ha hecho posible la configuración de un mercado de servicios basado en su tratamiento algorítmico. En efecto, el uso de estas herramientas estadísticas ha permitido introducir cambios radicales en la forma como las empresas planean y ejecutan sus estrategias publicitarias, motivando un creciente interés por el análisis de los patrones de comportamiento (behavioural targeting) de los usuarios de la red. ${ }^{1}$

Frente a este panorama de innovaciones radicales y ante la necesidad de preservar el libre flujo de la información como medio fundamental que facilite su recolección, almacenamiento y disponibilidad, se han venido proponiendo diferentes enfoques que implican una revisión de nociones jurídicas de rango constitucional, tales como el respeto a la privacidad, la libertad de expresión y el acceso a la información, las cuales han adquirido nuevos matices en el contexto de la sociedad de la información.

1 De acuerdo con Bergsten, Hufbauer y Miner (2014, pág. 190), "unconstrained data flows are responsible for tremendous economic gains. The McKinsey Global Institute calculated that open data could generate as much as $\$ 3$ trillion more in global income annually, in just seven sectors of the world economy (...) Open data are available without restrictions; are fundamental to new business models, from analysis of big data to cloud computing; and drive innovation, especially in small businesses."
Aunque el alcance de las discusiones actuales pone en evidencia la preocupación por la protección de los derechos fundamentales de los individuos frente al uso comercial de sus informaciones privadas, la preocupación subyacente a todos los esfuerzos regulatorios parece ser la de impedir que la intervención del Estado -y la influencia desmesurada de la sociedad civilobstaculicen la dinámica de un sector promisorio y en plena expansión.

El presente documento hace énfasis en la comprensión de los elementos y actores principales que conforman la economía digital, para a partir de allí avizorar nuevos ámbitos de protección de la privacidad con base en las premisas de la economía comportamental, y así establecer la medida en que los elementos teóricos de esta disciplina pueden llegar a informar enfoques basados en la confianza racional de los usuarios/ consumidores hacia los agentes del mercado respecto a su privacidad.

Desde esta perspectiva la reflexión contenida en el artículo se ha estructurado de la siguiente manera: en la primera parte se presenta una síntesis de los elementos que hacen parte de la discusión sobre la economía digital y su incidencia en el derecho a la privacidad. En la segunda parte se abordan los enfoques regulatorios así como las propuestas sobre autorregulación privada liderada por la industria. En una tercera parte se expone el estado actual de la economía digital en Colombia a partir del fallo que declaró la exequibilidad de la ley de protección de datos personales. En la cuarta parte se plantean algunas vías de inserción de la economía comporta- 
mental como medio para superar las fallas regulatorias detectadas en la parte tres, al tiempo que se presentan algunas consideraciones que podrían eventualmente mejorar la regulación con sujeción a las especificidades del ordenamiento jurídico colombiano.

\section{ESTADO ACTUAL DE LAS DISCUSIONES SOBRE REGULACIÓN DE LA PRIVACIDAD EN INTERNET}

Cualquier análisis sobre la situación de la protección de la privacidad en el contexto de la economía digital deberá partir de la incidencia que el tratamiento algorítmico de datos ha logrado en las estrategias de comercialización. Desde esta perspectiva, las cuestiones que surgen del tratamiento de la información personal exigen una delimitación del problema, que tome como punto de partida la imposibilidad de que sea el propio mercado el que regule los procesos por medio de los cuales se colecta, se transforma o se genera la información.

Aunque en la actualidad la mayoría de los países han implementado marcos regulatorios que solucionan de manera coyuntural los problemas que han ido surgiendo en el ámbito de la privacidad, en los países de referencia, principalmente EE. UU. y los países que conforman la Unión Europea (UE), el tratamiento de los datos continúa siendo conflictivo. Razón por la cual, nuevas voces postulan una lectura renovada y más cosmopolita de la protección de los datos personales, que tenga en cuenta tanto la amplitud de las innovaciones tecnológicas como su capacidad para introducir efectos disruptivos en la esfera del ejercicio de los derechos. Autores como Morozov (2014) plantean la necesidad de abordar el problema de cara a los desafíos actuales y futuros, lo cual implica ir más allá del análisis del alcance de las normas actuales, para adentrarse en la forma como los usuarios/ individuos perciben y emplean la tecnología, y de esta manera concebir instrumentos normativos que permitan interiorizar el ejercicio de los derechos en nuevos ambientes donde la aplicación específica de estos tiende a depender de entornos cibernéticos.

\section{A. Rasgos principales de la economía digital}

Como fenómeno global, los altos niveles de penetración de Internet han dado origen a una materia prima intangible que, a su vez, sirve de fundamento a todo un nuevo sector de la economía. Gutiérrez-Rubi (2014), señala cómo en efecto:

La velocidad con la que crecen los datos es vertiginosa. Se estima que la cifra se dobla cada dos años y según el Ericsson Mobility Report, su tráfico creció un $60 \%$ entre el segundo trimestre de 2013 y el de 2014. En 2020, habrá 50.000 millones de conexiones de personas, procesos, datos y objetos en Internet.

A pesar de que no se trata de un fenómeno reciente, ${ }^{2}$ las implicaciones derivadas de las ac-

2 El surgimiento de ciertos problemas regulatorios como resultado de la expansión de Internet y la difusión de su uso dentro de la sociedad fue advertido por Lad y Caldwell (2009, pág. 14), quienes ya en 2009 proponían algunas soluciones de política pública a nivel internacional: "The Internet is a globally fragmented industry with a technology 
tividades de recolección y almacenamiento de estas informaciones solo comenzaron a evidenciarse a finales de la década anterior, a partir del interés de las grandes empresas del sector de la publicidad y el mercadeo en línea (advertising networks) por lograr un acceso consolidado a estos grandes volúmenes de datos. Específicamente, el patrón de comportamiento de estas empresas comenzó a ser llamativo para los reguladores de Estados Unidos y Europa cuando se detectó una tendencia hacia la integración vertical entre quienes recopilaban información en línea de los usuarios (website publishers), y las otras empresas dedicadas a ofrecer servicios de tratamiento de datos (advertising networks).

En uno de los casos emblemáticos, la Comisión Europea abordó este tema como una cuestión transversal al proceso de integración entre dos empresas dominantes en el mercado de las redes de publicidad online; al hacerlo, planteó el ámbito de esta actividad en los siguientes términos:

The Parties submit that one of the rationales of the Transaction is to develop its activity in so-

that is growing in exposure geometrically. Further, it is only partially understood by those responsible for legislation. Calls by government for industry involvement in standards over privacy and security are evident in both Europe and the US. While initial technical standards in regulating HTML and XML languages on the Internet were developed democratically, the self-regulatory process in issues of encryption, privacy and taxation have not been as promising. Not only are a number of US and international regulatory bodies vying for jurisdiction, over 11 by one account, but no major trade group has emerged to mobilise the industry. Despite a recent FTc (Federal Trade Commission) sweep of 1,200 websites checking their privacy policy, the urgency to act has not hit the critical stage. This area represents a decisive opportunity for international cooperative policy with a mix of marketplace issues, technology expertise, consumer concerns and oversight organisations all in the dialogue." called "big data" analytics. This relates to the process of examining large amounts of data of a variety of types ("big data") to uncover patterns, correlations and other useful information. The primary goal of big data analytics is to help companies make better business decisions by enabling data scientists and other users to analyse large volumes of transaction data as well as other data sources that may not be assessed by conventional business intelligence programs. (Case сомP/M.7023 - PUBLICIS/ OMNICOM).

A partir de la aproximación asumida por la Comisión en este caso, resulta posible esbozar algunos de los rasgos predominantes de la economía digital que, desde luego, serán relevantes a la hora de referirnos a los enfoques que debería asumir una regulación apropiada para este sector en países en desarrollo como Colombia.

En primer lugar, el hecho de que la actividad declarada como principal en el marco del proceso de integración sea el big data analytics, pone de presente una de las particularidades de la economía digital que consiste en que su materia prima es la información, la cual fluye a través de la red permitiendo, simultánea o sucesivamente, a múltiples usuarios utilizarla para diversos propósitos. Al configurarse este intercambio dinámico entre quienes publican información, quienes la colectan o la almacenan y quienes la procesan con fines de convertirla en conocimiento con valor específico en el mercado, se logran nuevas interacciones de las cuales cada uno de los intervinientes extrae, o cree extraer, una utilidad o maximizar su propio beneficio. 
Así mismo, la cualificación que implica la referencia a "grandes y variados volúmenes de información (large amounts of data of a variety of types) pone de presente los efectos que se derivan del carácter de bien no rival que se atribuye a la información. Este atributo implica que la información colectada y almacenada por un agente del mercado es susceptible de ser utilizada en múltiples ocasiones para múltiples propósitos, sin que por ello se reduzca su valor para otros usuarios potenciales. Así, la información adquiere un valor en el mercado por el simple hecho de haber sido colectada, y a partir de allí, la creación de valor agregado respecto de esta dependerá de la posibilidad que se tenga para almacenarla o combinarla con otros tipos de información, esto es, de aplicar herramientas analíticas que deriven de tal información un conocimiento útil para algún agente que lo demanda dentro del mercado.

El escenario donde se desenvuelven estas interacciones está, a su vez, caracterizado por un vertiginoso cambio tecnológico y ciclos de innovación rápidos, los cuales se han visto estimulados por el surgimiento de esquemas de gestión estratégica de derechos de propiedad intelectual, a través de los cuales se protegen las herramientas (algoritmos) desarrolladas por las empresas (generalmente pequeñas), las cuales constituyen el pivote sobre el cual grandes corporaciones apalancan con su músculo financiero no solo el almacenamiento y procesamiento dinámico, sino la recolección de grandes volúmenes de información, permitiendo el diseño posterior de nuevas estrategias y modelos de negocio.
La consolidación de estas estructuras empresariales de base tecnológica y la posibilidad de que adquieran una posición de dominio de escala transnacional, suscita entonces una serie de interrogantes respecto de la tendencia hacia la preferencia por enfoques de autorregulación privada transnacional para este sector (impulsadas con insistencia por el sector privado), tal y como se analizará en la parte tercera. Con base en esta comprensión, surge la imperativa necesidad de configurar una base argumentativa que permita recobrar o mantener para el Estado niveles de intervención adecuados, de manera que se garantice un despliegue de todo el potencial técnico a estas aplicaciones no solo para estimular el mercado, sino también para integrarlas al cumplimiento de las funciones públicas, ${ }^{3}$ con lo cual, a su vez, se estaría garantizando la posibilidad de tutelar de manera técnica los derechos fundamentales que pueden ser objeto de vulneración como consecuencia de la expansión de este mercado.

\section{B. Actores de la economía digital}

Para comprender la forma como funciona este mercado es necesario igualmente partir de una descripción de los actores que participan y del entorno donde concurren, pues es de esta manera como se logra una mejor comprensión de

3 "El gobierno móvil. La gestión profunda del big data público nos permitiría una concepción móvil del servicio e información públicos. Multiformato, multipantalla y multicultural, sin concesiones. On y off integrados. La tecnología ya no es una elección sino una obligación para interactuar con los ciudadanos. Esta realidad cambia las ecuaciones de los formatos, las tipologías y los resultados de las relaciones entre usuariosciudadanos y las administraciones públicas, otorgando a los primeros un nuevo protagonismo." (Case сомP/M.7023 - PUBLICIS/OMNICOM). 
Ios efectos que las actividades de estos agentes pueden activar en la esfera de la privacidad.

En primer lugar encontramos a un anunciante (advertiser) que desea difundir un mensaje publicitario propio, mediante la publicación de su sitio web en una plataforma administrada por un editor de medios digitales (website Publisher). Esta primera interacción supone la fijación de unas condiciones de este respecto al uso o disponibilidad de medios tecnológicos que le permitan al anunciante hacer un seguimiento o valorar la eficacia de su publicidad mediante el monitoreo de la actividad de los consumidoresobjetivo.

La forma común mediante la cual el anunciante busca ampliar y fidelizar a sus usuarios es a través de los denominados cookies, los cuales ofrecen al usuario la ventaja de una identificación ágil -automática- en futuras transacciones. Para tal efecto, el editor administra estos mecanismos colectando el conjunto de informaciones que los usuarios han aceptado instalar en sus dispositivos personales, logrando vincular aspectos tales como gustos, preferencias, ubicación, inclinaciones o preferencias por medios de pago, etc. La información así colectada puede, a su vez, ser objeto de almacenamiento en repositorios o en las bases de datos de los propios editores, por periodos de tiempo y condiciones convenidas (ex-ante o ex-post) con los usuarios a través de las denominadas "políticas de privacidad".

Como tercer actor, y quizás el más relevante, encontramos a los denominados proveedores $u$ operadores de redes de publicidad (advertising networks), los cuales colectan y hacen disponible la publicidad ofrecida por miles de editores, con la particularidad adicional de que cuentan con la capacidad de reconocer o individualizar a los usuarios que acceden a su red de publicidad. Es justamente este rasgo, el que los habilita para ofrecer a los anunciantes la posibilidad de contar con perfiles detallados de sus usuarios y, de esta manera, hacer más asertiva la publicidad futura con base en los perfiles comportamentales resultado del análisis analítico de la información que han procesado.

Finalmente, el rol de usuario puede ser visto como el de un cuarto actor que accede a la información y confronta la decisión racional de hacer disponible su propia información personal, como medio de contribuir a la optimización de las funcionalidades de los servicios que le son ofrecidos a través de Internet. De esta manera no solo produce información, sino que, como fruto de su interacción, se convierte simultáneamente en receptor de aquella que ya ha sido procesada y puesta a su disposición por otros agentes dentro del mercado.

Es precisamente la profundización de la interacción de los actores, la que plantea la necesidad de una tutela a la privacidad en el entorno digital como emanación propia de la protección de los derechos fundamentales. Entendiendo dicha protección de la forma dinámica como lo hacen Anzit, Tato y Profumo (2010, pág. 73), es decir:

Como un viejo principio de derecho común, pero (...) en continua redefinición para fijar los 
límites de su protección, puesto que en un comienzo el derecho de la propiedad se aplicaba a las tierras y al ganado. Más tarde vino un reconocimiento a la naturaleza individual de la persona, a sus sentimientos y a su intelecto, extendiendo el derecho de propiedad a las cosas tangibles e intangibles, considerando el derecho a la vida como el derecho a disfrutar de ella, el derecho a ser dejado solo (the right to be left alone).

Esta observación resulta relevante en la medida en que ilustra la importancia de lograr que los marcos regulatorios en materia de protección de la privacidad que se conciban en el contexto de la economía digital estén por fuera, o si se quiere más allá, de la lógica tradicional (propicia para entorno analógico pero insuficiente en la era digital) que prevé la elaboración de reglas generales y abstractas con vocación de perdurabilidad, ${ }^{4}$ pues nada obsta para pensar en nuevos actores o tecnologías que incursionen en la dinámica del mercado afectando nuevamente la legitimación activa de los individuos en la defensa de sus derechos.

\section{Problemas que surgen con el advenimiento y consolidación de la economía digital}

Una vez establecida la noción de big data o flujo intensivo de datos como piedra angular de la economía digital, es fácil colegir que el presupuesto de base para la consolidación y crecimiento de este sector es el libre flujo de información en Internet. Esta proposición, aunque pudiera parecer obvia, ha ido encontrando interesantes matices a medida que la actividad de los individuos ha asumido gradualmente un carácter simultáneo de consumidor-productor de información (prosumers), ${ }^{5}$ lo cual permite que la información colectada por un anunciante se convierta en un bien inmaterial transable que circula en un mercado paralelo, invisible para los titulares de la información que está siendo transada.

Un ejemplo de esta doble condición la ilustra la posibilidad que actualmente ofrecen los sistemas de prestación de servicios médicos que, haciendo uso de la información colectada sobre los hábitos de consumo de sus afiliados, pueden generar perfiles orientados a la optimización de la prestación de tales servicios. ${ }^{6}$ Sin embargo, la

5 La autoridad europea para la protección de los datos personales European Data Protection Supervisor [EDPS] (2014a, pág. 2), propone un interesante símil: "Economic actors in the digital industry have been termed 'prosumers' who produce as well as consume information; individuals leave permanent traces across cyberspace, like silkworms depositing 'silk' which is then 'farmed' for different purposes by public and private organisations."

6 "Your Hospital Knows Your Secrets" (2014, pág. 21), "many patients and their advocates are voicing concerns that Big Data's expansion into medical care will threaten privacy. «It is one thing to have a number I can call if I have a problem or question ; it is another thing to get unsolicited phone calls». 
forma como se ha de colectar dicha información está sujeta a la expresión del consentimiento previo e informado por parte de tales afiliados, lo cual en últimas puede limitar el uso comercial futuro que se pueda obtener de dicha información. Es decir, la forma como se plantee el ejercicio de los derechos por parte del editor de la información puede terminar por disuadir al titular, quien podrá ejercer una reserva en cuanto al uso de estos y, por esa vía, excluir del mercado determinadas informaciones -las que considere de su esfera íntima-, limitando el ámbito de actividad de las empresas que se dedican al tratamiento y procesamiento de dicha información en etapas posteriores.

De esta manera, la solución a los problemas que surgen de la convergencia entre los derechos de los usuarios con los intereses de anunciantes y empresas dedicadas al procesamiento de la información disponible en Internet supone la reafirmación de la existencia de un conjunto de derechos, cuyo alcance se viene redefiniendo con el desenvolvimiento extraordinario de la economía digital. Esta dimensión es capturada adecuadamente por Sarra (2011, pág. 192), quien afirma con razón:

El surgimiento de la sociedad de la información globalizada puso en evidencia la existencia de dos derechos en pugna, el derecho a la libertad de expresión (y, genéricamente, el derecho a la información) y el derecho a la privacidad (y, por extensión, derecho sobre la información), aunque no es posible otorgar a ninguno de estos principios fundamentales preeminencia sobre el otro. Estos merecen protección en tanto derechos subjetivos puesto que no parece razonable proteger un derecho por el derecho mismo. Por ello, deben limitarse recíprocamente las prerrogativas fundamentales que pudieran entrar en conflicto.

En efecto, la reivindicación del libre flujo de información, como premisa fundamental que habilita las actividades en línea, parece encontrar rápidamente límites cuando los usuarios se ven confrontados a la ocurrencia de potenciales abusos por parte de agentes del mercado que, aprovechando su posición de dominio, pueden hacer uso indebido, inapropiado o extralimitado de la información personal o sensible a la que tienen acceso.

Resolver estos problemas resultaría relativamente fácil si se tratara de agentes económicos situados dentro de un mismo territorio y sometidos a una misma jurisdicción. Sin embargo, como se ha explicado, la economía digital debe su desarrollo, entre otros factores, a la concentración de las actividades intensivas en conocimiento y capital en manos de un grupo de empresas que han desarrollado la capacidad de procesar grandes volúmenes de información a escala transnacional, afectando positiva o negativamente los derechos de porciones cada vez más grandes de la población, a través de acciones que no necesariamente se concretan al territorio donde se ubican los afectados.

Desde esta perspectiva global, el carácter territorial de la regulación por parte de los Estados puede hacer aún más complicado el panorama, en la medida en que la protección del derecho a la intimidad se plantee mediante el recurso a 
medidas internas de corte "proteccionista" que, a su vez, pueden dar lugar a que los inversionistas busquen en estas fundamento para alegar presuntas violaciones a sus inversiones.

Por otro lado, hasta este punto, el panorama de tensión entre la capacidad económica y tecnológica de actores dominantes para imponer condiciones contractuales a los usuarios sin mayor posibilidad de intervención del Estado pareciera solucionarse por sí mismo, en la medida en que la afectación de la privacidad tiende a ser soportada/consentida por los propios usuarios, quienes llegan a considerarla como un mal menor o como un efecto colateral aceptable frente a los beneficios que se reciben en contraprestación.

Sin embargo, en la realidad, la situación precaria de los derechos fundamentales de los usuarios, especialmente del derecho a la privacidad, ha venido generando una desconfianza que, más allá de las consideraciones sobre la protección de los derechos del individuo, puede inclusive llegar a afectar el funcionamiento del mercado en el largo plazo, en la medida en que las soluciones normativas que propongan los Estados terminen adicionado imprevisibilidad a las conductas futuras de los actores, llegando inclusive a incidir en los costos de transacción. ${ }^{7}$

7 Acquisti (2010) presenta un detallado análisis de los aspectos económicos que circundan la protección de los datos personales en el entorno digital.

\section{PROPUESTAS REGULATORIAS EN TORNO A LA PRIVACIDAD}

Como se ha visto, la necesidad de restablecer o reparar la confianza de los usuarios respecto del manejo que las empresas agregadoras pueden hacer de sus informaciones sensibles es un elemento fundamental para el funcionamiento armonioso de la economía digital. Para tal fin, se han venido motivando al interior de las administraciones nacionales, una serie de procesos de elaboración concertada de normas destinadas a proveer los criterios para que los actores reguIen sus intereses dentro del mercado. En dicho panorama se destacan varios enfoques:

\section{A. El enfoque de la Federal Trade Commission (FTC)}

De manera general se puede afirmar que este enfoque se caracteriza por su carácter utilitario, el cual se refleja en el énfasis por consolidar una confianza de los consumidores que garantice, de parte de estos, el suministro continuo de la información, asegurando de manera simultánea la existencia de unas garantías mínimas a través de mecanismos de autorregulación concebidos e implementados por los procesadores/ agregadores de la información.

La evolución de las discusiones en la materia se divulga a la opinión pública a través de una serie de reportes y memorias de encuentros, en los cuales se recogen las propuestas efectuadas desde diversos niveles y por diferentes interesados en la regulación del manejo de datos 
y la privacidad de los usuarios (FTC, 2012). A pesar del carácter blando de las recomendaciones contenidas en tales discusiones, estas han permitido poner de relieve la existencia de temas sensibles, los cuales, a su vez, han ameritado acciones concretas en materia legislativa. ${ }^{8}$

Los tres componentes principales que actualmente exhibe este enfoque recogen la evolución en materia de protección de la privacidad en el entorno analógico y su vinculación con la protección del consumidor adaptándolos a las especificidades del big data. El primero de ellos hace énfasis en la necesidad de incorporar mecanismos de protección de la privacidad desde la etapa de diseño del producto (concept of privacy by design), ${ }^{9}$ lo que implica clarificar la posición de los actores que colectan y comparten información respecto de la gestión de aspectos tales como la seguridad de la información, los límites en cuanto a la cantidad de información que pueden colectar, así como las restricciones en cuanto a la retención, retiro y fidelidad de las informaciones a las que tienen acceso.

El segundo componente promueve la implementación de mecanismos que simplifiquen la elección que los usuarios pueden hacer respecto del uso de sus informaciones, lo cual está relacio-

8 "Since the Commission issued the preliminary staff report, Congress has introduced both general privacy bills and more focused bills, including ones addressing Do Not Track and the privacy of teens. DoNot-Track Online Act of 2011, S. 913, 112th Congress (2011); Do Not Track Me Online Act, H.R. 654, 112th Congress (2011)".

9 "The concept of privacy by design includes limitations on data collection and retention, as well as reasonable security and data accuracy. By considering and addressing privacy at every stage of product and service development, companies can shift the burden away from consumers who would otherwise have to seek out privacy- protective practices and technologies" (FTC, 2012, pág. 2). nado con la oportunidad que estos tienen para expresar su consentimiento para no ser objeto de monitoreo comportamental (behavioral targeting), mediante la instalación de archivos de texto (cookies) que permiten identificar o singularizar la actividad del usuario en la red. En la medida en que el enfoque privilegia la instalación de tales archivos basado en un consentimiento tácito informado, surgen dudas sobre la efectividad de la protección propuesta, puesto que la instalación no consentida del cookie, en sí misma, implica una invasión de la privacidad del usuario. Es por esta razón que en paralelo se promueve la utilización de mecanismos de do not Track, ${ }^{10}$ como una manera de garantizar la posibilidad para los usuarios de excluir de la red aquellas informaciones que el propio usuario considere sensibles.

El tercer componente es complementario de los anteriores y consiste en aumentar la trasparencia en las actividades de quienes colectan, almacenan y procesan información, lo cual implica el deber correlativo de informar al usuario sobre la posibilidad que tiene de acceder a su información colectada, conocer la finalidad para la cual ha sido colectada o procesada y, si es del caso, ejercer su derecho a retirar la información considerada sensible. Por esta vía se busca, por un lado, incrementar la comprensión de los usuarios sobre el tratamiento que se hace de sus informaciones y, por el otro, fomentar las buenas prácticas que reduzcan la conflictividad

10 Estos mecanismos utilizan una señal de transmisión de un usuario, la cual es activada a través de un ícono que el consumidor asocia con las políticas de protección de su privacidad. Las discusiones en torno a dicho mecanismo se centran en la oportunidad para hacer uso de este (opt-in, o opt-out). 
en el ámbito digital, y por consiguiente permitan la consolidación de la economía digital a largo plazo.

\section{B. El enfoque de la Unión Europea}

La diferencia fundamental en la aproximación de la Unión Europea (UE) con el enfoque de la FTc está dada por la existencia de un principio de protección activa de los derechos fundamentales, que emana del Tratado de Lisboa y que sitúa la protección de tales derechos como una prioridad de la actividad regulatoria de los Estados de la Unión, lo cual incluye obviamente la regulación de la privacidad en Internet. ${ }^{11}$ Bajo esta premisa, los órganos rectores envían varias señales a las administraciones nacionales para que estas a su vez comprendan las líneas generales de la protección de la privacidad y las adapten a sus prioridades de promoción del sector de la economía digital.

En términos generales es posible afirmar que los órganos encargados de ejercer un control sobre la actividad de los actores de la economía digital - la Corte de Justicia de la Unión Europea y la Comisión- parecen estar decantándose por intervenciones de tipo económico, sustentadas

11 Hustinx (2013, pág. 18) señala cómo "the entry into force of the Lisbon Treaty in December 2009 had an enormous impact on the development of EU data protection law. (...) In the first place, the Charter was given the same legal value as the Treaties in Article 6(1) of the Treaty on European Union (TEU). It thus became a binding instrument, not only for the EU institutions and bodies, but also for the Member States acting within the scope of EU law. The right to the protection of personal data was moreover specifically mentioned in Article 16(1) of the Treaty on the Functioning of the European Union (TFEU) among the general principles of the EU. This meant that some of the main elements of Directive 95/46/EC have now reached the level of EU primary law. This is also relevant for the current reform, as we will see at a later stage". en la premisa de que a través de una política de competencia eficaz se disminuyen los riesgos derivados de la eventual integración vertical a la que es proclive el mercado del big data. ${ }^{12} \mathrm{De}$ esta manera, se podría afirmar que la ue reserva un rol regulador a los Estados miembros, por la vía de las normas de competencia y protección al consumidor, sin que ello excluya la posibilidad de enfoques autorregulatorios que permitan integrar nuevos elementos o soluciones concebidas tanto por expertos como por sectores de la sociedad civil. ${ }^{13}$

Aunque las preocupaciones en torno a la privacidad de los usuarios son esencialmente las mismas que en los Estados Unidos, la evolución de las discusiones se encuentra liderada por un ente administrativo creado por la Directiva 95/46/CE del Parlamento Europeo y del Con-

12 Así se puede colegir del siguiente aparte de la decisión sobre la Integración Microsoft-Yahoo: "There are currently only three relevant independent technical platforms for intermediation of search ads (or closely related text-based contextual ads, which operate on an international level: Microsoft, the Yahoo search business and Google. The proposed transaction technically removes one advertising platform. Even though the combining platforms currently service a very low number of publishers in the EEA, this could theoretically harm publishers because it may result in reduced competition between the remaining advertising platforms and consequently lower prices paid for publishers' web space" (Case No COMP/M.5727 - Microsoft/ Yahoo!, párr. 233). [Notas de pie de páginas eliminadas de la cita].

Recientemente la Comisión Europea (2014) anunció la creación de una Asociación Público-Privada (APP) destinada a la promoción del uso del big data, lo cual incluye participar en las discusiones sobre el tema de los posibles impactos en la privacidad de los usuarios. "La APP en materia de datos es una asociación entre la Comisión Europea y Big Data Value Association, una organización sin ánimo de lucro, dirigida por la industria, entre cuyos miembros están ATC, IT Innovation, IBM, SINTEF, la Universidad de Bolonia (CINI), la Universidad Politécnica de Madrid, NOKIA Soluciones y Redes, THALES, la Universidad de DuisburgoEssen, Siemens, SAP, Engineering, TIE Kinetix, ANSWARE, Software AG, Orange, Atos, INDRA, ITI, VTT, Fraunhofer, DERI y la Universidad Técnica de Berlín. La asociación está abierta a las empresas y organizaciones de investigación que deseen unirse a ella" (Comisión Europea, 2014, pág. 2). 
sejo de 24 de octubre de $1995,{ }^{14}$ denominado "Grupo de protección de las personas en lo que respecta al tratamiento de datos personales", el cual ha producido una serie de informes sobre la situación actual y las condiciones para potenciar el crecimiento de la economía digital, con preeminencia del respeto por los derechos de los usuarios. ${ }^{15}$

\section{Los enfoques de autorregulación}

Uno de los rasgos comunes que se puede extraer del análisis conjunto de los enfoques previamente abordados es una sutil hostilidad hacia la intervención del Estado por la vía de la regulación, a la cual se le atribuye el nocivo potencial de inhibir la innovación y por consiguiente el desarrollo del sector. Dicha actitud parece estar fundamentada igualmente en preocupaciones relacionadas con el impacto de ciertas medidas que, implementadas por parte de los Estados en el ámbito interno, puedan llegar a tener algún impacto en el despliegue de las inversiones proyectadas por empresas interesadas en ofrecer estos servicios, así como respecto del control de actividades por parte de operadores de redes de publicidad más allá de las fronteras donde prestan sus servicios. Esta posición es claramente defendida por el National Foreign Trade Council (2011, pág. 1) cuando señala:

14 Relativa a la protección de las personas físicas en lo que respecta al tratamiento de datos personales y a la libre circulación de estos datos (Unión Europea, 1995).

15 Véase al respecto: European Commission. Dictamen 2/2010 sobre publicidad comportamental en línea, adoptado el 22 de junio de 2010. Disponible en: http://ec.europa.eu/justice_home/fsj/privacy/index_ en.htm (última consulta 31 de octubre de 2014).
Digital protectionism is a growing threat around the world. A number of countries have already enacted or are pursuing restrictive policies governing the provision of digital, commercial and financial services, technology products, or the treatment of information to favor domestic interests over international competition. Even where policies are designed to support legitimate public interests such as national security or law enforcement, businesses can suffer when those rules are unclear, arbitrary, unevenly applied or more trade restrictive than necessary to achieve the underlying objective. What's more, multiple governments may assert jurisdiction over the same information, which may leave businesses subject to inconsistent or conflicting rules.

Esta dimensión transnacional de la economía digital plantea un serio desafío de cara a su armonización regulatoria, máxime cuando la complejidad de temas como la privacidad o la protección del consumidor pueden conducir a los tecnócratas encargados a plantear regulaciones regresivas en términos tanto de garantía del acceso a la información como del respeto de la privacidad. Es en este contexto donde se viene planteando la conveniencia de la autorregulación privada transnacional (TPR, por sus siglas en inglés) ${ }^{16}$ como mecanismo para garantizar la pertinencia técnica, económica, legal y ética de las regulaciones en el ámbito de la economía digital. Autores como Caffagi, (2010, pág. 6) reco-

16 Caffagi (2010, pág. 23) define la TPR como "a new body of rules, practices and processes, created primarily by private actors, firms, NGOs, independent experts like technical standard-setters and epistemic communities, either exercising autonomous regulatory power or implementing delegated power, conferred by international law or by national legislation." 
nocen el surgimiento de estos esquemas en el contexto del cambio tecnológico, planteándolo con el siguiente alcance:

Another factor contributing to the growth of TPR is the development of new technologies that redistribute rule-making power in favor of private actors and transform the role of the nation state. ICт and, in particular, Internet regulation provides an illustration of the role of technology in shifting rule-making power from national to transnational and from public to private. In fact, the characterizing feature is that of hybridity. The conflict and the subsequent agreement between Google and the People's Republic of China (PRC) highlights new modes of regulation at the global level based on contracts between multinational firms and states.

En este escenario surge el interrogante sobre hasta qué punto es deseable o conveniente confiar la regulación sobre los aspectos relacionados con la encriptación y la privacidad de la información en la red a grupos de expertos, pues siguiendo al mismo Cafaggi:

The definition of experts has changed over time and in some areas of expertise has become much less hierarchical. In the field of Internet governance, the diffusion of epistemic communities self-regulating themselves has bloomed, giving rise to a multiplicity of non-profit organizations or informal networks. In this model the rules are mainly technical; the regulator is a private non profit organization, supposedly independent from the industry and from the final beneficiaries but often subject to capture. The regulator differs from the regulated and from the beneficiaries and its legitimacy is based on expertise (2010, pág. 11).
Aunque las reservas que se puedan adelantar frente a estas aproximaciones son bastante pertinentes, los enfoques autorregulatorios ofrecen ventajas respecto a la disponibilidad de la información técnico-legal esencial para informar el debate jurídico, sobre todo en los países en desarrollo, donde uno de los problemas fundamentales es la dificultad de lograr que la actividad regulatoria efectuada por las autoridades logre hacer que los individuos/consumidores comprendan e interioricen las externalidades en materia de afectación de sus derechos fundamentales que surgen de sus interacciones en el entorno digital.

\section{PERSPECTIVAS REGULATORIAS PARA COLOMBIA}

Un análisis de la regulación de la economía digital en Colombia es indisociable de la política comercial que se ha venido consolidando en la última década. En efecto, la fragmentación normativa que caracteriza la regulación del comercio internacional es un rasgo prominente que se extiende también a la economía de los datos. De acuerdo con la observación que hacen Bergsten, Hufbauer y Miner (2014), este factor debería servir también como incentivo para el diseño de marcos regulatorios que permitan la armonización de los servicios digitales en el contexto de la regionalización de los intercambios comerciales. En opinión de dichos autores: "Rules governing cross-border data flows are incomplete and therefore bilateral and regional agreements present an opportunity to create a framework for open data and to push for constructive policies" (pág. 190). 


\section{A. Panorama general de las} normas actuales

Desde esta perspectiva es posible afirmar que la regulación actual ${ }^{17}$ corresponde a un enfoque que procura, de manera primordial, asegurar la inserción de Colombia dentro del sector del big data, configurando un entorno normativo orientado al estímulo de inversiones por parte de empresas multinacionales del sector. Esta desafortunada percepción, a su vez, se traduce en claros vacíos ${ }^{18}$ que generan dificultades en cuanto a la protección de los derechos fundamentales de los usuarios, planteando serias dudas respecto del cumplimiento de las premisas económicas que justifican la existencia de un régimen normativo de la protección de datos.

Esta situación afecta por extensión el diseño de estrategias (políticas públicas) encaminadas a vincular intereses públicos y privados en torno al procesamiento de datos, retardando la consolidación de este nuevo sector económico y su capacidad de apalancar la actividad innovadora de pequeñas y medianas empresas. ${ }^{19} \mathrm{En}$

17 Ley 1266 de 2008 y Ley 1581 de 2012 y sus decretos reglamentarios.

18 En este sentido comparto la opinión expresada en el salvamento de voto a la sentencia C-748/11: "En contrario, la legislación estatutaria omite esa etapa en razón de un trasplante normativo acrítico e incompleto del estándar europeo de protección de datos personales, preocupado exclusivamente en lograr la uniformidad legislativa que facilite la oferta de servicios empresariales de tratamiento de datos transmitidos desde el exterior, pero en abierto detrimento y contradicción del ámbito de vigencia del habeas data que prescribe la Carta Política. A este respecto, advertimos que la normatividad estatutaria desconoció abiertamente la diferenciación de las distintas etapas en la gestión de datos personales que está prevista en la Constitución, asunto que, como se ha indicado, no estaba delegado al arbitrio del legislador, sino que está sometido a un marco de referencia, de raigambre constitucional y suficientemente preciso."

19 Aparicio (2004, pág. 473), destaca la forma en que "los Estados pueden actuar sobre el mundo virtual tanto en forma indirecta -so- este sentido, resulta pertinente el examen de uno de los salvamentos de voto a la sentencia C-748/11 - en la que se declaró la exequibilidad de la Ley 1581 de 2012-, donde se ponen en evidencia los serios vacíos en la efectividad de la protección consagrada en dicho dispositivo normativo:

Con todo, la norma estatutaria objeto de análisis en la sentencia C-748/11, en una adopción mecánica de la legislación extranjera sobre habeas data, en particular el estándar europeo de protección de datos personales, redujo los actores a tres: el titular, el responsable del tratamiento y el encargado del mismo. De acuerdo con esa clasificación, se imponen deberes de protección del derecho al habeas data a la persona que adopta las decisiones sobre la base y/o tratamiento de los datos (responsable del tratamiento), al igual que sobre la persona que adelanta, a instancia del responsable del tratamiento, el proceso técnico de administración de datos (encargado del tratamiento).

\section{(...)}

Esta división, en nuestro criterio, es inconstitucional al generar un déficit de protección del derecho al habeas data. Si se observa con detenimiento la legislación estatutaria avalada por la mayoría, se tiene que no existe una regulación sobre los deberes de las fuentes, y especialmente respecto de los usuarios del dato

metiendo a sus propios nacionales a concretas reglas para el acceso tanto a la actividad empresarial en la Red como a su simple disfrute o utilización con fines no comerciales, o participando en la elaboración de la tecnología que constituye la naturaleza de Internet, fomentando que sea más o menos abierta- como directa, en ámbitos de incursión muy concretos y claros, en los cuales su intervención resulta incluso obligada, con el fin de proteger concretos intereses, como puede ser la propia dignidad de la persona y, en especial, de los menores de edad o la protección de contratante débil." [Cursivas añadidas]. 
personal. Es decir, esta normatividad parte de considerar que la obligación de protección del derecho al habeas data se agota en el proceso de administración de los datos personales, que realizan el responsable y el encargado del tratamiento. Por ende, parte de un supuesto contraevidente, consistente en que los datos son acopiados y tratados, mas no recopilados y posteriormente circulados a los usuarios. Esto en contravía con el mandato constitucional contenido en el artículo 15 C.P., consistente en que la cláusula general de libertad y demás garantías consagradas en la Carta son predicables no solo de la etapa de tratamiento, sino también en la recolección y la circulación de datos personales.

Es claro que al haberse autorizado el aludido trasplante normativo con desconocimiento o imprecisión acerca de aspectos fundamentales, como lo son la determinación de los sujetos que intervienen en el ciclo de tratamiento algorítmico de la información, la Corte no solo ha dificultado la realización de un derecho fundamental, ${ }^{20}$ que asume características específicas en nuestra condición de país emergente, sino que, además, ha introducido un factor de inseguridad jurídica que no puede de ninguna manera ser entendido como un estímulo a la inversión en el sector.

Ahora bien, aunque la existencia de un marco normativo no implica la solución instantánea de los múltiples problemas que plantea la protección de la privacidad en entornos tecnológicos caracterizados por innovaciones de carácter

20 Constitución Política, artículo 15. "En la recolección, tratamiento y circulación de datos se respetarán la libertad y demás garantías consagradas en la Constitución." incremental, la reflexión contenida en el salvamento de voto pareciera acertar al poner de presente la necesidad de enfocar la discusión desde una perspectiva más amplia, que permita capturar el fenómeno económico subyacente, el cual está relacionado, en buena medida, con lo que la doctrina autorizada denomina "la paradoja de la protección de la privacidad", la cual consiste en la ausencia de correspondencia entre actitudes (algunas veces exageradas) y comportamientos (frecuentemente laxos o despreocupados) de los individuos al momento de tomar decisiones que pueden afectar el ejercicio de sus derechos fundamentales en el entorno digital. ${ }^{21}$

\section{B. Espacio para la economía comportamental en la protección al derecho a la privacidad}

Aunque el salvamento de voto de la sentencia analizada no lo haga explícito, a la luz de la realidad actual su contenido puede ser interpretado como un llamado urgente a la revisión legislativa de la regulación vigente, de manera que se permita estructurar una tutela de la privacidad a partir de la interiorización de los deberes de los individuos y por ende de la importancia que estos asignan a sus derechos.

Esto supondría un esfuerzo legislativo para fundamentar la nueva regulación en el estudio de las decisiones racionales, tal como se propugna dentro de la disciplina de la economía compor-

\footnotetext{
21 Una interesante discusión al respecto es ofrecida por Hoofnagle y Urban (2014), quienes retoman los conceptos desarrollados por Alan Westin, en su obra Privacy and Freedom (1967).
} 
tamental. Un tal enfoque podría aportar importantes elementos que permitan compatibilizar los estímulos a la economía digital como sector económico con los desarrollos legales y jurisprudenciales que con anterioridad a la sentencia venía desarrollando la Corte Constitucional, pero sobre todo de cara a los comportamientos actuales del consumidor medio colombiano. ${ }^{22}$

Aunque son múltiples y variados, uno de los aportes fundamentales a la relación entre la teoría de la elección racional y la protección de la privacidad en entornos digitales está contenido en los trabajos de Acquisti y Grossklags (2007). Del punto de partida de sus investigaciones es posible extraer los objetivos básicos que deberían orientar la regulación, lo cual toma especial relevancia en el contexto socioeconómico de los países emergentes, como Colombia, en donde las acciones gubernamentales han promovido la exposición progresiva de los ciudadanos a las nuevas tecnologías como medio para mejorar la inclusión social. ${ }^{23}$ En palabras de los mencionados autores:

Privacy is a complex decision problem resulting in opinions, attitudes, and behaviors that differ

22 Sentencia C-748/11 "En efecto, la jurisprudencia constitucional ha precisado que las características de los datos personales —en oposición a los impersonales - son las siguientes: "i) estar referido a aspectos exclusivos y propios de una persona natural, ii) permitir identificar a la persona, en mayor o menor medida, gracias a la visión de conjunto que se logre con el mismo y con otros datos; iii) su propiedad reside exclusivamente en el titular del mismo, situación que no se altera por su obtención por parte de un tercero de manera lícita o ilícita, y iv) su tratamiento está sometido a reglas especiales (principios) en lo relativo a su captación, administración y divulgación." [Cursivas en el original].

23 En tal sentido comparto la anotación de Martínez (2010, pág. 116) en el sentido de que "Pueda que hoy, el analfabetismo funcional que se da en el mundo digital, o el deseo de experimentar con los nuevos servicios, faciliten la tarea a quienes antepongan el beneficio inmediato a la privacidad de los usuarios. Pero ni es una situación que se mantendrá en el tiempo, ni resultaría tolerable que así fuera." substantially from one individual to another. Subjective perceptions of threats and potential damages, psychological needs, and actual personal economic returns all play a role in affecting our decisions to protect or to share personal information. Thus inconsistencies or even contradictions emerge in individual behavior: sometimes we feel entitled to protection of information about ourselves that we do not control, and end up trading away that same information for small rewards. Sometimes we worry about personal intrusions of little significance, but overlook those that may cause significant damages. (...) privacy choices are affected by incomplete information and in particular asymmetric information: data subjects often know less than data holders about the magnitude of data collection and use of (un)willingly or (un) knowingly shared or collected personal data; they also know little about associated consequences. (...) the complex life-cycle of personal data in modern information societies can result in a multitude of consequences that individuals are hardly able to consider in their entirety (...) even with access to complete information and cognitive power to process it exhaustively, various behavioral anomalies and biases could lead individuals to take actions that are systematically different from those predicted by rational choice theory. (Acquisti y Grossklags, 2007, pag. 1).

De acuerdo con esta observación, corresponde ahora entrar a analizar los escenarios en los cuales se puede dar la integración de los conceptos de la economía comportamental a las discusiones sobre la privacidad. En este sentido, la discusión queda planteada en torno a: i.) los efectos que la libertad de mercado puede generar en los derechos de la colectividad, noción vin- 
culada al espacio regulatorio con que el Estado debe contar para garantizar el libre ejercicio de los derechos fundamentales de los individuos; $y$ ii) la definición de un ámbito para el despliegue de la iniciativa privada en torno a nuevos sectores económicos. Zuiderveen (2006) plantea los contornos de este problema regulatorio en torno a la protección de la privacidad aclarando parcialmente las razones que justifican la intervención del Estado en lo que a priori sería una esfera reservada al individuo:

A basic starting point in economics is that contractual freedom is desirable, because completely free trade should lead to the highest social welfare. But there may be reason for government intervention in markets under rational choice theory. The possible grounds for limiting contractual freedom can be roughly divided in two categories. First, sometimes third parties suffer costs resulting from a contract: externalities. Second, sometimes contract parties have difficulties entering a contract in their best interests, because of information asymmetries or transaction costs for instance. (Pág. 27).

Esta reflexión es necesaria por cuanto ilustra la forma como se viene dando una modificación de los fundamentos con base en los cuales los individuos interpretan el alcance de sus derechos y su efectividad en ámbitos tales como la salud, la alimentación, el trabajo o la privacidad (en el caso de la economía de los datos). En efecto, la súbita disponibilidad de una gran variedad de nuevas tecnologías y la existencia de nuevos entornos para el ejercicio de los derechos fundamentales han venido generando la falsa sensación de que tales derechos pueden ser prescindibles o renunciables, abriendo la posibilidad de que algunos agentes dentro del mercado exploten, de manera desequilibrada, esa deficiente percepción. ${ }^{24}$

Ahora bien, la capacidad de discernir los efectos (externalidades) que la disposición de los derechos puede tener en el contexto de la sociedad de la información nos conduce a reflexiones más profundas, pues en la medida en que perfilemos la protección de los derechos sobre la base de las capacidades cognitivas de los individuos es posible que al mismo tiempo estemos abriendo paso a una división, poco deseable, entre individuos capaces de asumir posiciones críticas como consumidores, dando preponderancia a la protección de sus derechos como condición sine qua non para el desarrollo de la economía digital, mientras que otra parte de la población - la mayor parte- no se encuentra en capacidad de comprender los efectos que sus comportamientos, en dicho entorno, generan para el ejercicio de sus derechos. Esta preocupación es presentada por Luhmann (1997), para quien:

The worst imaginable scenario might be that the society of the next century (XXI) will have to accept the metacode of inclusion/exclusion. And this would mean that some human beings will be persons and other only individuals; that some are included into function systems for (successful or un successful) careers and

24 En tal sentido resulta pertinente la intervención de Rossoglou (2014), quien enfatiza correctamente sobre cómo lo que está en discusión en materia de privacidad en Internet "son derechos personales, derechos que deben ser retenidos por los individuos respecto de sus datos personales. Este concepto de derechos individuales incorporado en el derecho europeo por más de 20 años no debe cambiar. La discusión entonces debe ser sobre cómo cumplir y no sobre cómo modificar dicho derecho". 
others are excluded from these systems, remaining bodies that try to survive next day.

Consecuencialmente, si tanto el acceso a Internet como el desarrollo de medios cibernéticos han terminado por convertirse en aspectos críticos para la realización de ciertos derechos fundamentales, resulta igualmente legítimo y conveniente abordar la regulación de la economía digital desde una perspectiva comportamental, es decir, centrando los objetivos regulatorios en la modulación de las externalidades que se derivan de la tendencia a la concentración del poder de mercado por parte de los agregadores, así como en la evidente incapacidad de la mayoría de individuos para discernir los efectos que se derivan de la disponibilidad de sus informaciones personales en el entorno digital.

\section{La intervención del Estado en materia de big data}

De manera general, el problema de la intervención del Estado en los mercados suele asociarse con aumentos de los costos de transacción como resultado de la relativa ineficiencia estatal para controlar eventuales externalidades negativas. Para el caso de la economía de los datos, el incremento en los costos en los que las empresas agregadoras deberán incurrir en procura de mejorar la transparencia de sus actividades parece no justificar dicha intervención estatal, sino que suele ser apreciado como una forma de perturbar el funcionamiento de un mercado en el cual la seguridad e intangibilidad de los derechos de los usuarios se han venido prote- giendo por la vía de la negociación privada de los actores intervinientes.

Sin embargo, vale la pena reiterar que hasta hace muy poco el mercado de los datos funcionó explotando de manera ostensible las asimetrías de información, logrando configurar un escenario en el cual pocos eran los consumidores capaces de asumir una posición crítica o informada sobre sus decisiones, mientras que la mayoría se mostraba más bien desinteresada o pragmática con respecto al uso de sus datos personales, llegando incluso a considerar que la afectación a la intimidad se da como algo colateral o accesorio, que no amerita mayor preocupación. Esta situación se ha visto agravada frecuentemente por posiciones gubernamentales que, como en el caso de Colombia, privilegian arbitrariamente una aproximación mercantilista de la protección de los datos personales.

\section{Acciones puntuales}

Un punto de partida para orientar correctamente la discusión sobre el rol del Estado en el despliegue de la economía digital es determinar con claridad las oportunidades que surgen para el país en relación con un mercado global caracterizado por sus externalidades en red, esto es, un mercado en el cual se da preponderancia al tamaño consolidado de todo el mercado y no al tamaño individual de las empresas que a él puedan concurrir. En este escenario surge la inquietud sobre si ¿existe verdaderamente la posibilidad de que empresas nacionales (locales) puedan competir a nivel global en el mercado del procesamiento intensivo de datos? 0 , si sim- 
plemente ¿estamos llamados a aportar nuestra información personal como esa nueva materia prima, que permite el despliegue de las actividades de las empresas agregadoras, incluso fuera de las fronteras de nuestro territorio?

En este sentido es posible afirmar que existen dos formas a través de las cuales el Estado puede simultáneamente contribuir a la consolidación de la economía digital y corregir algunas de sus externalidades negativas. Por un lado, está la participación del propio Estado dentro del sector a través de la implementación progresiva de herramientas de gobierno digital, ${ }^{25}$ y por el otro, está la capacidad del Estado para estimular la innovación en el sector, mediante la inversión estratégica en I+D que asegure la participación de la comunidad científica nacional en la solución de problemas comunes de los países emergentes frente a los desafíos que plantea la expansión de la economía digital.

En el primer caso es claro que muchas de las funciones que contemporáneamente son atribuidas al Estado -aun a pesar del avance y preponderancia del discurso neoliberal- se ejecutan mediante la recolección de información sensible; tal es el caso de los registros disciplinarios, de antecedentes judiciales, ciertos tipos de información médica o migratoria, así como todo el volumen de información contenido en los re-

25 Para Gutiérrez-Rubi (2014): "El gobierno móvil. La gestión profunda del big data público nos permitiría una concepción móvil del servicio e información públicos multiformato, multipantalla y multicultural, sin concesiones. On y off integrados. La tecnología ya no es una elección sino una obligación para interactuar con los ciudadanos. Esta realidad cambia las ecuaciones de los formatos, las tipologías y los resultados de las relaciones entre usuarios-ciudadanos y las administraciones públicas, otorgando a los primeros un nuevo protagonismo." gistros contables de empresas, que finalmente son colectados y procesados en el contexto de la actividad fiscalizadora o de otras inherentes a la actividad estatal. Respecto de estos temas, curiosamente la posición de la ciudadanía frente al manejo de sus datos o informaciones personales suele generar una mayor preocupación, tal vez debido a la posibilidad que existe de asignar una responsabilidad al Estado por los daños que este pueda causar a los particulares en ejercicio de sus funciones; asociación que no se da respecto de las empresas que igualmente y de manera aún más agresiva pueden acceder o procesar esas mismas informaciones.

Es precisamente allí donde el Estado puede lograr que las actitudes de los ciudadanos respecto del uso público que dan a sus datos personales se extiendan hacia el ámbito privado, pues en la medida en que la utilización de los datos, hecha por las entidades públicas, se haga bajo criterios que la ciudadanía pueda fácilmente extrapolar a sus relaciones cotidianas con agregadores privados, esa misma comprensión tendrá necesariamente que irradiar la capacidad cognitiva de los ciudadanos cuando actúan como consumidores dentro del mercado.

Con respecto al estímulo a la innovación y a la I+D dentro de este sector, es relevante recordar que aún en el caso de las economías avanzadas como la de Estados Unidos, los beneficios de la extraordinaria expansión de la economía digital no han logrado contribuir a aliviar la pérdida de la competitividad en el mercado global. ${ }^{26}$

26 Mazzucato (2013, pág. 177) señala cómo "decades of government
investment in the science and technology base have made de US a 
Esta realidad impone un rol al Estado en cuanto a la fijación de las prioridades en materia de I+D en el contexto de la economía digital, con miras a lograr la ampliación del mercado de servicios digitales mediante el estímulo a la investigación en lo relativo a protocolos novedosos que permitan aliviar algunas de las externalidades negativas que propicia la expansión de la economía digital. Esta posibilidad es alentada por Acquisti (2010), quien ha puntualizado al respecto:

The advance provided by research in cryptographic protocols is that privacy needs can be satisfied without significant damage to useful flows of personal data. Regulators' interventions aimed at fostering the dissemination and adoption of those technologies, therefore, may help us reach that more desirable economic equilibrium. In such a co-regulatory framework, economics could highlight different trade-offs, technology could help achieve more desirable equilibria, and regulatory intervention could nudge the market to adopt those technologies (pág. 19).

De esta manera queda claro que la intervención deseable del Estado es multidimensional, en el sentido en que debe conducir a la consolidación de este nuevo sector y su enorme potencial desde el punto de vista económico, propiciando la innovación pero vinculada a la implementación de marcos regulatorios que permitan a los países emergentes ubicar la protección de los derechos de los usuarios en el centro de las preocupaciones del mercado. En otras pala-

successful innovator, but have paradoxically failed to secure high levels of employment, to increase tax revenues and to promote export of good and services." bras, el mercado de las soluciones que deben explorar los países emergentes es aquel de las herramientas tecnológicas que garanticen verdaderamente el ejercicio de los derechos fundamentales de millones de habitantes, los cuales, hoy en día, creen erróneamente estar disfrutando de los beneficios de la era digital.

\section{CONCLUSIONES}

La economía digital y su enorme potencial ofrecen múltiples soluciones a las necesidades de miles de consumidores alrededor del planeta. La particularidad del análisis algorítmico está en lograr adaptar dichas soluciones a la medida de los propios gustos, preferencias o inclinaciones de los consumidores que, desprevenidamente, consienten en aportar sus informaciones. Sin embrago, este fantástico panorama se hace frágil ante la existencia innegable de importantes brechas tecnológicas y culturales, lo cual en últimas plantea serios desafíos para la consolidación de este nuevo sector de la economía global.

En este escenario el rol atribuido al Estado se ha visto desfigurado por una idealización del rol de las empresas multinacionales, al punto de que los enfoques de autorregulación privada transnacional aparecen a priori como los mecanismos que podrían dotar al mercado con las herramientas necesarias para su consolidación. A pesar de las ventajas que su implementación pudiera llegar a representar, es indispensable que las autoridades nacionales no cedan el núcleo duro de sus competencias en materia de protección de los derechos fundamentales de 
sus ciudadanos, pues de hacerlo, indirectamente estarían abdicando sus poderes soberanos, emanados del legítimo ejercicio democrático, al poder incontrolable que hoy parece asumir el capital y las empresas transnacionales que lo representan.

En el contexto colombiano, los preceptos y la jurisprudencia constitucional han desarrollado una base sólida sobre la cual el Estado puede plantearse el ejercicio de su poder regulatorio frente a este tema. Sin embargo, la normativa actual deja translucir la inmensa influencia de las empresas multinacionales del sector, al igual que la ausencia de un análisis legislativo crítico en torno a lo que representa el procesamiento algorítmico de la información en el contexto socioeconómico que enfrentan actualmente los países emergentes.

Con todo, la existencia del marco regulatorio actual supone el primer paso para que la sociedad civil se organice e interrogue sobre el alcance y justificación de un marco legal que controle el manejo que personas o empresas pueden hacer de informaciones sensibles que se han hecho disponibles por medios de electrónicos. Este elemento resulta crucial, pues es a partir de allí que deben construirse los cambios regulatorios que hoy demanda el sector.

El análisis económico del derecho, y en particular algunas aproximaciones desde la economía comportamental, aportan elementos nuevos al estudio de las reacciones de los individuos cuando se enfrentan a la toma de decisiones racionales en ambientes donde prevalecen asi- metrías en la información. Es claro que orientar las políticas de protección de privacidad con base en estos enfoques puede aparecer como algo ilusorio, más aún, cuando la expansión de la Internet ha permeado la sociedad en general, alimentando una serie de percepciones deformadas de lo que debe ser el acceso y disponibilidad de la información personal en la red.

Recomponer el rol de Estado por la vía de su inserción en la economía digital, mediante el uso progresivo e informado de estrategias de gobierno digital, es actualmente la mejor opción para avanzar hacia la socialización de los alcances que tiene el manejo algorítmico de datos para la sociedad en su conjunto. Esta sensibilización a través de la propia acción estatal le permitirá no solo conocer la dinámica y la forma como operan estas herramientas de tratamiento de la información, sino que, al mismo tiempo, contribuirá a que la sociedad pueda exigir a los actores privados con la misma intensidad que lo hace frente al Estado cuando se dan abusos o malos manejos a la información personal.

Por último, pero no menos importante, es que en cumplimiento de su misión el Estado logre promover la innovación tecnológica, mediante el estímulo a la I+D de nuevas aplicaciones que solucionen las externalidades negativas que se vayan detectando a medida que se consolide el avance de la economía digital. Una aproximación de cooperación sur-sur podría beneficiar esquemas de colaboración que permitan la investigación conjunta -por ejemplo, sobre protocolos criptográficos-, y con ello solucionar a corto plazo problemas que hoy son comunes a 
varios países emergentes, a través de la participación en el mercado de compañías ubicadas dentro de estos países.

\section{Referencias}

1. Acquisti, A. (2010). The Economics of Personal Data and the Economics of Privacy: 30 Years after the OECD Privacy Guidelines. Paris: OECD.

2. Acquisti, A., \& Grossklags, J. (2007). What Can Behavioural Economics Teach Us About Privacy? En A. Acquisti, S. Gritzalis, C. Lambrinoudakis y S. di Vimercati (Edits.), Digital Privacy: Theory, Technologies and Practices. New York: Auerbach Publications, Taylor and Francis Group.

3. Anzit, R., Tato, N. y Profumo, S. (2010). El derecho informático: aspectos fundamentales. Buenos Aires: Cathedra Jurídica.

4. Aparicio, J. (2013). Protección de los datos personales y redes sociales: un cambio de paradigma. En M. Moro, J. Aparicio y A. Batuecas, Autores, consumidores y comercio electrónico (págs. 141-157). Madrid: Editorial Colex.

5. Bergsten, F., Hufbauer, G., \& Miner, S. (2014). Bridging the Pacific: Toward Free Trade and Investment between China and the United States. Washington, District of Columbia: Peterson Institute for International Economics.
6. Cafaggi, F. (2010). New Foundations of Transnational Private Regulation. EUI Working Paper, Robert Schuman Centre for Advanced Studies. Florencia: European University Press.

7. Case COMP/M.5727 - Microsoft/ Yahoo! Search Business Notification of 15 January 2010 pursuant to Article 4 of Council Regulation No 139/2004. European Commission. SG-Greffe(2010) D/2118 C(2010) 1077

8. Case COMP/M.7023 - PUBLICIS/OMNICOM. Commission decision pursuant to Article 6(1)(b) of Council Regulation No 139/2004. Luxembourg: Office for Publications of the European Union.

9. Comisión Europea. (13 de octubre de 2014). Comunicado de prensa, La Comisión Europea y el sector de los datos ponen en marcha una asociación de 2500 millones de euros para lograr el dominio de los macrodatos. Obtenido de European Commission: http://europa.eu/rapid/press-release_IP14-1129_es.htm

10. Corte Constitucional de Colombia. Sentencia C-748 de 2011 (M. P.: Jorge Ignacio Pretelt Chaljub; octubre 6 de 2011).

11. European Commission. (s. f.). Dictamen 2/2010 sobre publicidad comportamental en línea. Adoptado el 22 de junio de 2010. Recuperado el 31 de octubre de 2014 de European Commission: http://ec.europa. eu/justice_home/fsj/privacy/index_en.htm 
12. European Data Protection Supervisor [edps]. (2014a). Report of workshop on Privacy, Consumers, Competition and Big Data. Recuperado el 31 de octubre de 2014 de edps: www.edps.europa.eu

13. European Data Protection Supervisor. (2014b). Preliminary Opinion of the European Data Protection Supervisor: Privacy and competitiveness in the age of big data: The interplay between data protection, competition law and consumer protection in the Digital Economy. Recuperado el 31 de octubre de 2014 de edps: www.edps.europa.eu

14. Federal Trade Commission [ftc]. (March, 2012). Protecting Consumer Privacy in an Era of Rapid Change: Recommendations for Businesses and Policymakers.

15. Gutiérrez-Rubi, A. (2014). Administraciones públicas y Big Data. Recuperado el 13 de octubre de 2014 de cincodias: http://cincodias.com/cincodias/2014/10/10/economia/1412963119_079817.html

16. Hoofnagle, C. y Urban, J. (2014). Alan Westin's Privacy Homo Economicus. 49 Wake Forest Law Review. Recuperado el 10 de enero de 2015 de scholarship: http://scholarship. law.berkeley.edu/facpubs/2395

17. Hustinx, P. (2013). EU Data Protection Law: The Review of Directive 95/46/EC and the Proposed General Data Protection Regulation. Recuperado el 12 de noviembre de 2014 de edps: www.edps.europa.eu
18. Lad, L. J., \& Caldwell, C. B. (2009). Collaborative Standards, Voluntary Codes and Industry Self-regulation. Journal of Corporate Citizenship, (35), 67-80.

19. Luhmann, N. (1997). Globalization on World Society? How to conceive a modern society. International Review of Sociology, 7(1), 6779.

20. Martínez, R. (2010). Protección de los datos personales y redes sociales: un cambio de paradigma. En A. Rallo y R. Martínez, Derecho y redes sociales (págs. 83-116). Pamplona: Thompson Reuters.

21. Mazzucato, M. (2013). The Entrepreneurial State: Debunking Public vs. Private Sector Myths. Londres: Anthem Press.

22. Morozov, E. (2014). The real privacy problem. MIT Technology Review. Recuperado el 10 de febrero de 2015 de MIT Technology Review: http://www.technologyreview.com/ featuredstory/520426/the-real-privacy-problem/

23. National Foreign Trade Council. (2011). Promoting Cross-Border Data Flows: Priorities for the Business Community. Recuperado el 29 de agosto de 2014 de nftc: http:// www.nftc.org/default/Innovation/PromotingCrossBorderDataFlowsNFTC.pdf

24. Rossoglou, K. (2014). 5th Annual European Data Protection \& Privacy Conference. Bruselas. Obtenido de eu-ems: http://eu-ems. 
com/summary.asp?event_id=234\&page_ id $=2016$

25. Sarra, A. V. (2000). Comercio electrónico y derecho. Aspectos jurídicos de los negocios en Internet. Buenos Aires: Editorial Astrea.

26. Unión Europea. (1995). Directiva 95/46/CE del Parlamento Europeo y del Consejo, de 24 de octubre de 1995, relativa a la protección de las personas físicas en lo que respecta al tratamiento de datos personales y a la li- bre circulación de estos datos. Diario Oficial de las Comunidades Europeas $\mathrm{n}^{\circ} \mathrm{L} 281$ de 23/11/1995 p. 0031 - 0050- DO L 281 de 23/11/1995 pp. 0031 - 0050.

27. "Your Hospital Knows Your Secrets". Bloomberg Newskeek, July 7-13.

28. Zuiderveen, F. (2013). Consent to Behavioural Targeting in European Law: What are the Policy Implications of Insights from Behavioural Economics? Amsterdam Law School Research Paper. 